IdeAs

Idées d'Amériques

$17 \mid 2021$

Villes et culture dans les Amériques

\title{
L'American Library Association et l'American Library in Paris : un patrimoine culturel centenaire
}

Cara S. Bertram

Traducteur : Anne Reynès-Delobel

OpenEdition

Journals

Édition électronique

URL : https://journals.openedition.org/ideas/11129

DOI : 10.4000/ideas. 11129

ISSN : 1950-5701

Éditeur

Institut des Amériques

Référence électronique

Cara S. Bertram, «L'American Library Association et l'American Library in Paris : un patrimoine culturel centenaire », IdeAs [En ligne], 17 | 2021, mis en ligne le 01 mars 2021, consulté le 07 juin 2021. URL http://journals.openedition.org/ideas/11129; DOl : https://doi.org/10.4000/ideas.11129

Ce document a été généré automatiquement le 7 juin 2021

\section{(c) $(1 \otimes \Theta$}

IdeAs - Idées d'Amériques est mis à disposition selon les termes de la licence Creative Commons Attribution - Pas d'Utilisation Commerciale - Pas de Modification 4.0 International. 


\title{
L'American Library Association et l'American Library in Paris : un patrimoine culturel centenaire
}

\author{
Cara S. Bertram
}

Traduction : Anne Reynès-Delobel

1 L'American Library Association (ALA) a vu le jour le 6 octobre 1876, au terme d'une réunion de trois jours, organisée dans le cadre du congrès des bibliothécaires (Conference of Librarians), l'année de l'Exposition du Centenaire à Philadelphie. Si elle est aujourd'hui reconnue comme la doyenne et la plus importante des associations nationales de bibliothécaires, l'ALA a connu des débuts modestes. Après 1917, sa mission sera de promouvoir les services de bibliothèque et d'information ainsi que la profession de bibliothécaire aux États-Unis. Toutefois, avant cette date, c'est une organisation locale que Burton Stevenson, bibliothécaire et romancier, décrit dans ces termes: "une association professionnelle comme il en existe tant d'autres, qui ronronne dans ses traditions et ses habitudes, et se préoccupe principalement de détails techniques » (Wiegand W., 1986:235).

2 L'entrée en guerre des États-Unis change la donne. Lors de son congrès annuel, à Louisville, dans le Kentucky, l'ALA décide de créer le Library War Service (LWS), un programme d'envergure financé par les pouvoirs publics, qui vise à fournir des livres et des services de bibliothèque aux soldats stationnés dans les camps d'entraînement aux États-Unis et à l'étranger. Le LWS est le programme de service public le plus ambitieux piloté par l'AlA à l'époque et sa réussite a de quoi étonner quand on sait que l'association compte alors à peine plus de 3300 membres.

3 Si plusieurs des projets lancés par l'ALA après-guerre n'ont pas connu le même succès, le LWS a eu des répercussions durables, notamment en matière de leadership et de service public. En témoigne en particulier l'American Library in Paris (ALP). De fait, pour comprendre comment l'ALA est devenue un service public et son engagement dans la bibliothéconomie internationale, il est essentiel de revenir sur sa participation 
bénévole à l'effort de guerre et le rôle qu'elle a joué dans la création et le maintien de l'American Library in Paris.

\section{Le Library War Service et l'American Library in Paris}

4 La mission première du LWS était de collecter des fonds et des livres auprès du public, de créer des bibliothèques dans les camps d'entraînement et de les doter en personnel, et de fournir des services de bibliothèque dans les hôpitaux militaires. Dès 1920, le LWS a recueilli 5 millions de dollars de dons du public, créé trente-six bibliothèques de camp et établi des bibliothèques dans plus de cinq cents lieux. Plus de mille bénévoles sont engagés dans l'opération.

Malgré l'importance accordée à la politique intérieure pendant la guerre, un nombre limité de représentants de l'ALA reçoivent l'autorisation de se rendre en France. Ils coopèrent avec d'autres services, tels que le YMCA et la Croix-Rouge, pour distribuer des livres aux soldats engagés sur le front. Les services de l'ALA en Europe, d'abord dirigés par McKendree Raney puis par Burton Stevenson, ne disposent pas de quartier général. Au cours de l'été 1918, l'ALA est invitée par le YMCA à partager des locaux au 10, rue de l'Elysée, ce qui permet à l'association de s'établir à Paris et, le 29 août 1918, d'ouvrir sa "Paris Library " qui sert à la fois de siège social à l'ALA en France, de bibliothèque pour les soldats et les résidents anglophones en France, et d'exemple du fonctionnement d'une bibliothèque publique américaine (Young A., 1981 : 66-67).

6 Après la signature de l'armistice, le Library War Service poursuit ses activités et d'autres bibliothécaires américains obtiennent leur visa pour l'Europe. La principale priorité de l'association reste d'assurer sa mission de service public en fournissant un soutien moral aux soldats stationnés dans les cantonnements et les hôpitaux militaires, en attente de leur démobilisation. Cette activité revêt plusieurs formes, de la plus simple (créer des salles lecture) à la plus complexe (élaborer des programmes éducatifs et d'apprentissage pour anticiper le retour des soldats à la vie civile). En juillet 1919, Mary Eileen Ahern, bibliothécaire et responsable des campagnes de promotion et de communication de l'ALA, rapporte qu'un soldat lui a confié que sans le secours des livres, il aurait perdu la raison (Ahern M. E., 1919 : 312).

7 Fin 1919, les opérations du LWS sont en phase de clôture. L'ALA transfère alors une grande partie des livres collectés et de ses activités au gouvernement fédéral américain. Reste la question de la "Paris Library » et de ses collections. Le public anglophone se montre en faveur du maintien de la bibliothèque. Dans le compte-rendu de l'un des congrès de l'ALA, le secrétaire de l'association, Carl Milam, note que «La bibliothèque de Paris va être maintenue, que nous le voulions ou non. La question intéresse, voire passionne, les hommes d'affaires américains et le public anglophone » («Special A. L. A. Conference », $1920: 4$ ).

8 Forte de ce soutien, l'American Library in Paris voit le jour en 1920. Son statut évolue vers celui d'une organisation indépendante, mais les liens avec l'ALA ne sont pas pour autant rompus. Ainsi, au cours des premières années d'existence de la bibliothèque, l'ALA va lui fournir une aide financière et un tiers des membres de son conseil d'administration. Si ces liens se sont distendus au fil du temps, l'ALA conserve un siège au conseil d'administration, signe tangible d'une relation désormais centenaire dont les archives de l'ALA conservent la mémoire. 


\section{Le Library War Service dans les archives de l'ALA} d'archives, abrités dans les archives de l'American Library Association, à l'Université de l'Illinois, à Urbana-Champaign. Cet ensemble se compose de lettres, rapports, publications, listes de livres, coupures de journaux, photographies et diapositives sur verre, scrapbooks et affiches. Arthur P. Young, l'auteur de l'historique du LWS, précise que les archives abritent près de trente mille pages de correspondance et dix mille coupures de journaux et de magazines relatives aux activités de l'ALA pendant la Première Guerre mondiale (Young A., 1981 : 115).

La valeur de cette collection réside dans sa diversité: dossiers administratifs, publicités, photographies et archives personnelles composent une histoire à la fois riche et complexe. La mise en œuvre du LWS n'est en effet pas allée sans heurts. Tandis que la presse vantait les exploits de l'ALA, la correspondance de ses dirigeants révèle la précarité qui entoure l'entreprise : peu de gens savent ce que recouvre l'acronyme de l'association, des conflits ponctuels l'opposent au YMCA ou à la Croix-Rouge pour déterminer à qui il revient de diriger telle ou telle opération ou de s'attribuer les mérites de telle ou telle autre. Des griefs exposés dans des courriers adressés au siège américain ou dans les correspondances privées portent sur la disparition de livres et de périodiques pendant le transport, ce qui retarde l'approvisionnement des bibliothèques destinées aux soldats.

11 Les archives des bénévoles de l'ALA permettent une approche plus personnelle du LWS. Dans les lettres adressées à sa famille, une bibliothécaire, Harriet Long, décrit en détail le travail qu'elle accomplit auprès des soldats cantonnés à la frontière avec le Mexique, ses efforts pour pallier le manque de main d'œuvre, les envois de livres qui s'égarent et autres difficultés qui, dit-elle, lui donnent l'envie «d'employer un langage tout à fait indigne de son sexe et de sa fonction» (Harriet C. Long Papers). Les documents personnels de Charles Greene décrivent qu'il s'est vu nommé en urgence bibliothécaire de référence à Camp Johnson, en Floride, puis, dans la foulée, bibliothécaire en chef par intérim et ce, avant même d'avoir mis les pieds sur le terrain, ce qui souligne la résilience et la capacité d'adaptation des bénévoles de l'ALA. Une lettre de Mary Eileen Ahern révèle qu'elle a aidé une famille à localiser la tombe de leur fils mort au combat en France, ce qui montre que son rôle excédait ses fonctions de bibliothécaire (Mary E. Ahern Papers). Grâce à ces récits et souvenirs personnels, les archives administratives du LWS acquièrent un souffle et une voix.

archives de l'ALA possèdent également une riche collection de documents promotionnels relatifs au LWS. Dans le cadre des campagnes de sensibilisation du public à la collecte de livres et de fonds, on a imaginé une série d'affiches aux couleurs vives. Pour inciter le public américain à donner des livres et des revues, les représentants de l'ALA aux États-Unis projetaient des dispositives montrant des photographies de soldats dans les bibliothèques des cantonnements ou des hôpitaux, à l'aide de lanternes magiques. Ces diapositives étaient également utilisées dans les cantonnements pour promouvoir les services de bibliothèque. Dans le cadre des campagnes de sensibilisation du grand public à l'effort de guerre du LWS, une série de cartes postales montrant l'envoi de livres, les bibliothèques de camp et des soldats blessés en train de lire a également été éditée. 

sont en cours de numérisation par le personnel des archives en collaboration avec le Digitization Services Unit de l'Université de l'Illinois. Ainsi, l'ensemble des diapositives sur verre et des cartes postales a été scanné et on peut facilement y accéder en ligne. Les affiches de la Première Guerre mondiale ont également été numérisées et sont disponibles sur demande. Les scrapbooks du LWS ont, quant à eux, fait l'objet d'un travail de restauration et ils sont en cours de numérisation en vue d'être rendus accessibles en ligne. également accessible en ligne, notamment les « Caroline Webster Papers ». Les archives Webster représentent une ressource documentaire inestimable sur les débuts des services bibliothécaires dans les établissements hospitaliers. On projette désormais de poursuivre la numérisation des archives textuelles, notamment celles du « War Service Correspondence, 1917-1923 », qui comprend quarante-sept volumes reliés de lettres et de rapports du LWS.

\section{Localiser l'American Library in Paris dans les archives de l'ALA}

15 Si les archives du LWS forment un seul sous-ensemble au sein des archives de l'ALA, celles relatives à l'ALP ne sont pas aussi bien organisées. Elles ne sont en effet pas classées par sujet, mais par créateur ou par provenance. Par exemple, les documents provenant du LWS forment un seul groupe. Toutefois, étant donné que l'ALP n'est pas un bureau ou une branche de l'ALA, localiser des informations sur l'ALP dans les archives de l'ALA peut demander des recherches minutieuses.

recherches permettent de savoir quels bureaux et unités de l'ALA ont pu collaborer ou échanger avec l'ALP. Des classeurs et les documents portant sur l'ALP se trouvent dans les archives de plusieurs unités de l'ALA, notamment celle du LWS, du bureau des relations internationales ("ALA International Relations Office»), du bureau du directeur général («Executive Director's office»), de la présidence («ALA Presidents »), dans les archives relatives aux membres et affiliés, ainsi que dans des publications de l'ALA qui remontent aux origines de l'ALP. La dissémination de ces documents dans les archives de l'ALA témoigne de l'étroitesse des liens qui unissent les deux institutions.

Trouver des informations sur l'ALP dans les dossiers du LWS peut se révéler étonnamment difficile. Ainsi, il est utile de se rappeler que la bibliothèque ne s'appelait pas initialement l'American Library in Paris. Nombre de rapports, discours et lettres y font référence diversement : «Paris Library ", «ALA Headquarters in Paris » (siège de l'ALA à Paris), « ALA Headquarters in France » (siège de l'ALA en France) ou simplement « Headquarters Library » (bibliothèque du siège).

Les documents relatifs aux origines de l'ALP au temps du LWS se trouvent dans la vaste collection du "War Service Correspondence ", organisée dans le cadre des activités de l'ALA en France (War Service Correspondence). La consultation du Bulletin of the American Library Association, publication officielle à l'ALA jusqu'en 1938, permet également d'obtenir des informations sur l'ALP au cours de ses premières années, sous forme d'articles et de rapports. De manière plus surprenante peut-être, qui s'intéresse 
aux origines de l'ALP trouvera dans les actes du congrès annuel de l'ALA une autre riche source d'information. Ainsi, les actes organisés pendant la Grande Guerre fournissent des rapports détaillés des activités de l'ALA écrits de la main de ses représentants et dirigeants, des évaluations de ces activités, ainsi que les transcriptions des discussions et des discours. Les informations afférentes à la création de l'ALP en tant que société morale se trouvent dans les actes du congrès annuel de l'ALA en 1920. De nombreux documents conservés dans les archives de l'ALA se rapportent à l'histoire de l'ALP pendant l'entre-deux-guerres et la Seconde Guerre mondiale. La majeure partie des archives du bureau des relations internationales de l'ALA ayant trait à l'ALP couvrent les années 1920 à 1945. Une série de documents sur la correspondance entre le bureau des relations internationales et l'ALP comprend des lettres à et de Jessie Carson, Edward Sumner, Milton Lord et H.M. Lydenberg, entre autres. Une autre série comprend des brochures, cartes postales et publications faisant la promotion des services de l'ALP et retraçant son histoire. Les dossiers de l'American Legion Conference en 1927 comprennent notamment des lettres, mémorandums et brochures concernant l'aide apportée par l'ALA à l'ALP, à l'occasion de l'American Legion Paris Conference.

Les archives du bureau du directeur exécutif de l'ALA constituent une autre source d'information sur l'histoire de l'ALP pendant l'entre-deux-guerres et la Seconde Guerre mondiale («American Library in Paris Correspondence, 1922-1945»). Cette série composée de trois boîtes contient la correspondance fournie des directrices de l'ALP, Dorothy Reeder et Clara de Chambrun, qui donnent toutes deux un aperçu du travail de la bibliothèque pendant l'Occupation. Les archives d'Althea $\mathrm{H}$. Warren («Althea $\mathrm{H}$. Warren Papers»), présidente de l'ALA de 1943 à 1944, livrent également des informations supplémentaires sur les activités de l'ALA pendant la Seconde Guerre mondiale et ses relations avec l'ALP.

21 Une autre piste de recherche est l'éphémère Paris Library School de l'ALP, fondée en 1923. Placée sous la direction de la bibliothécaire et secrétaire adjointe de l'ALA, Sarah Bogle, et parrainée par le Comité américain pour les régions dévastées (CARD), cette école avait pour mission de former les bibliothécaires aux méthodologies américaines. Des difficultés financières ont néanmoins contraint à sa fermeture en 1929. Ce dossier est constitué de quatre boîtes de correspondance, rapports, listes d'étudiants et rapports budgétaires.

22 Les archives de Foster E. Mohrhardt donnent une idée des liens étroits entre l'ALA et l'ALP après-guerre. Mohrhardt a siégé au conseil d'administration de l'ALP de 1965 à 1973. Ses documents contiennent des procès-verbaux de réunions, des dossiers de projets et une proposition de déménager l'ALP dans d'autres locaux, datée de 1973.

Toutefois, la preuve de la relation de longue date entre les deux organisations se trouve peut-être dans les comptes rendus et les minutes des réunions du conseil et du bureau exécutif de l'ALA. Lors de la réunion de l'hiver 2020, le Conseil de l'ALA a ainsi voté et approuvé une résolution félicitant l'ALP à l'occasion de son centenaire ${ }^{1}$.

Une partie de la correspondance du directeur général («Executive Director's American Library in Paris Correspondence ») est numérisée et accessible en ligne, tout comme la série complète sur l'American Legion Conference. Une partie du dossier « ALP Pamphlet and Publication » a été également numérisée et est accessible aux chercheurs. D'autres ressources, peut-être moins évidentes, sont également disponibles au format numérique, notamment les actes des congrès de l'ALA, ainsi que les minutes de son 
conseil d'administration et de son conseil. En dépit du faible pourcentage d'archives numérisées relatives à l'ALP, l'intérêt des chercheurs s'est accru à l'occasion du centenaire de la bibliothèque. Ce regain d'intérêt nous a conduit à envisager de numériser davantage de documents, notamment la correspondance et les dossiers du bureau du directeur général et du bureau des relations internationales portant sur l'ALP.

\section{BIBLIOGRAPHIE}

Ahern, Mary Eileen, “A. L. A. News from Overseas,” Bulletin of the American Library Association, vol. 13, n 3, July 1919.

“Special A. L. A. Conference," Bulletin of the American Library Association, vol. 14, n 1, January 1920.

Thomison, Dennis, A History of the American Library Association, 1876-1972, Chicago, American Library Association, 1978.

Wiegand, Wayne A., The Politics of an Emerging Profession: The American Library Association, 1876-1917, New York, Greenwood Press, 1986.

Young, Arthur, Books for Sammies: The American Library Association and World War I, Pittsburgh, Beta Phi $\mathrm{Mu}, 1981$.

\section{NOTES}

1. La transcription de cette discussion est conservée dans les archives de l'ALA.

\section{AUTEURS}

\section{CARA S. BERTRAM}

Cara S. Bertram, diplômée de la Western Washington University, est responsable du service d'archives à l'Université de l'Illinois à Urbana-Champaign. Elle est chargée de la gestion des archives de l'American Library Association et du service de référence pour les archives de l'Université de l'Illinois. cbertra@illinois.edu 